\title{
Accelerator-Based Fusion with a Low Temperature Target
}

\author{
R. E. Phillips and C. A. Ordonez \\ Department of Physics, University of North Texas, Denton, Texas 76203-5017
}

\begin{abstract}
Neutron generators are in use in a number of scientific and commercial endeavors. They function by triggering fusion reactions between accelerated ions (usually deuterons) and a stationary cold target (e.g., containing tritium). This setup has the potential to generate energy. It has been shown that if the energy transfer between injected ions and target electrons is sufficiently small, net energy gain can be achieved. Three possible avenues are: (a) a hot target with high electron temperature, (b) a cold non-neutral target with an electron deficiency, or (c) a cold target with a high Fermi energy. A study of the third possibility is reported in light of recent research that points to a new phase of hydrogen, which is hypothesized to be related to metallic hydrogen. As such, the target is considered to be composed of nuclei and delocalized electrons. The electrons are treated as conduction electrons, with the average minimum excitation energy being approximately equal to $40 \%$ of the Fermi energy. The Fermi energy is directly related to the electron density. Preliminary results indicate that if the claimed electron densities in the new phase of hydrogen were achieved in a target, the energy transfer to electrons would be small enough to allow net energy gain.
\end{abstract}

Keywords: Fusion Energy, Neutron Generator, Electronic Energy Loss

PACS: 52.40.Mj, 28.52.Av,29.25.Dz,89.30.Jj

\section{INTRODUCTION}

One objective of fusion research is to achieve fusion reactions through some artificial means that generates as much fusion energy as input energy. This elusive "break even" point has been sought for more than sixty years. The large majority of such efforts have centered around increasing the temperature and pressure of fusion fuel as plasma to favorable regimes. This requires an incredible amount of input energy which is prone to rapid loss.

A tangential use for fusion systems that cannot achieve "break even" is as sources of neutron flux. Neutron generators are in use in a number of scientific and commercial endeavors. They function by triggering fusion reactions between accelerated ions (usually deuterons) and a stationary cold target (e.g., containing tritium). This setup has the potential to generate energy, provided energy losses can be minimized. By far the largest energy loss by the incident ions is to target electrons. Associated collisions generate no energy and slow the incident ions, lowering the probability that subsequent collisions will produce a fusion reaction. It has been shown that if the energy transfer between injected ions and target electrons is sufficiently small, net energy gain can be achieved. Three possible avenues are: (a) A hot target with high electron temperature [1-3]. In this case, the electrons are moving so much faster than the incident ions that the charged particles have very little time to interact with each other and less energy is lost. (b) A cold non-neutral target with an electron deficiency [4]. With fewer electrons in the target, fewer collisions occur and less energy is lost. However, this method would face formidable interdisciplinary issues [5]. (c) A cold target with a high Fermi energy. The electron plasma becomes partially transparent to the incident ions when ions cannot transfer enough energy to excite a portion of the electrons. A study of the third possibility is reported in light of recent research. Holmlid et al. have provided experimental evidence for an ultra-dense phase of protium and deuterium referred to as " $\mathrm{P}(-1)$ " and "D(-1)," respectively [6-10]. During the experiments, the $\mathrm{D}(-1)$ and $\mathrm{P}(-1)$ states were observed using time-of-flight measurements. A laser pulse would ionize electrons within the ultra-dense hydrogen. Adjacent nuclei would then be strongly repelled from one another, and a "Coulomb explosion" would result. By assuming the particles start effectively at rest, and recording the energy each particle possessed, the nuclei separation was calculated. A mass density of $1.4 \times 10^{8} \mathrm{~kg} / \mathrm{m}^{3}$ and

Application of Accelerators in Research and Industry

AIP Conf. Proc. 1525, 94-96 (2013); doi: 10.1063/1.4802297

(C) 2013 AIP Publishing LLC 978-0-7354-1148-7/\$30.00 
electron density of $10^{35} \mathrm{~m}^{-3}$ was reported for $\mathrm{D}(-1)$. The hypothesized method of generation is via the collapse of small quantities of metallic-like Rydberg matter into clusters that are emitted from a porous potassiumdoped iron oxide catalyst. Neither $\mathrm{P}(-1)$ nor $\mathrm{D}(-1)$ have been synthesized in bulk. Also, the properties of $\mathrm{P}(-1)$ and $\mathrm{D}(-1)$ have not been independently confirmed.

The target is considered here to be composed of nuclei and delocalized electrons. An expression for $F_{e}$, a fusion energy gain factor, has two parts: the energy lost to electrons in the system, and the energy produced by fusion reactions. The gain factor is defined simply as power produced divided by power lost.

\section{Energy Loss}

For the loss term, we first obtain an expression for the stopping power. Since we assume the electrons are delocalized, they form a degenerate plasma. The plasma is treated as conduction electrons in the low temperature limit. The Fermi energy is

$$
E_{\mathrm{Fermi}}=\frac{\hbar^{2}}{2 m_{\theta}}\left(3 \pi^{2} n_{\theta}\right)^{2 / 3} \text {. }
$$

Here, $\hbar$ is Plank's constant divided by $2 \pi, n_{e}$ is the electron density and $\mathrm{m}_{\mathrm{e}}$ is the mass of an electron. Notice that the Fermi energy increases with the electron density. The average minimum excitation energy is

$$
\mathrm{U}=\mathrm{E}_{\mathrm{Fermi}}-<\mathrm{E}>=(2 / 5) \mathrm{E}_{\mathrm{Fermi}},
$$

where $<\mathrm{E}>=(3 / 5) \mathrm{E}_{\mathrm{Fermi}}$ is the average energy for conduction electrons. The stopping cross section $\mathrm{S}$ for the case of a stationary target is [11]

$$
S=\frac{2 \pi \mathrm{K}_{c}^{2} e^{4} m_{\text {incident }} \lambda}{E_{\text {in cident }} m_{\text {target }}} \text {. }
$$

Here, $\mathrm{K}_{\mathrm{c}}$ is the Coulomb force constant, e is the charge of each particle, which is assumed to be the charge of a proton, $m$ is the mass of the respective particles as labeled, $\mathrm{E}_{\text {incident }}$ is the incident beam particle energy, and

$$
\lambda=\ln \left[\sqrt{\left.1+\frac{4 M_{\text {reduced }}^{2} V_{\text {incident }}^{4}}{U^{2}}\right]} .\right.
$$

Here, the reduced mass is between an incoming ion and a target electron, and the minimum excitation energy is replaced by its average. The stopping power is

$$
\frac{\mathrm{dE}_{\mathrm{loss}}}{\mathrm{dl}}=\mathrm{Sn}_{\theta}
$$

\section{Energy Gain}

The gain term is

$$
\frac{\mathrm{dE}_{F}}{\mathrm{dl}}=n_{\mathrm{ion}} \sigma_{f} E_{f} \text {. }
$$

Here, $\mathrm{E}_{\mathrm{f}}$ is the fusion energy produced by one reaction, $\mathrm{n}_{\text {ion }}$ is ion density, and $\sigma_{\mathrm{f}}$ is the fusion cross section. Our gain term divided by our loss term assuming a neutral target, $\mathrm{n}_{\text {ion }}=\mathrm{n}_{\mathrm{e}}$, is

$$
F_{\theta}=\frac{E_{f} \sigma_{f}}{S}=\frac{E_{f} E_{\text {incident }} m_{\theta} \sigma_{f}}{2 \pi \mathrm{e}^{4} K_{c}^{2} m_{\text {incident }} \lambda}
$$

\section{Results}

Figure 1 shows a plot of the fusion energy gain factor as a function of the target's electron density, considering tritons to be incident on a deuterium target. 


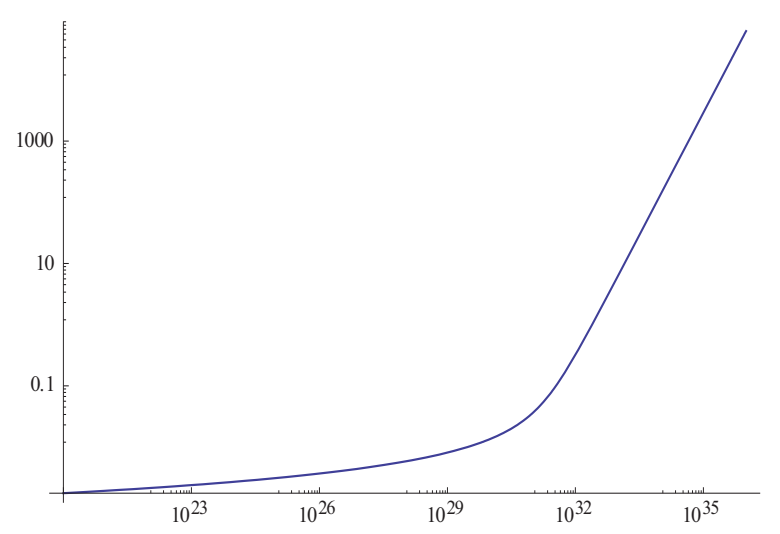

FIGURE 1. Plot of the energy gain factor for a given electron density. Tritons are incident on a deuterium target.

The parameter values used for the plot in Fig.1 are as follows:

The peak fusion cross section is used for a $\mathrm{D}+\mathrm{T}$ reaction, $\sigma_{\mathrm{f}}=5 \times 10^{-28} \mathrm{~m}^{2}$. The energy generated from one $\mathrm{D}+\mathrm{T}$ reaction is $\mathrm{E}_{\mathrm{f}}=17.6 \mathrm{MeV}$. The beam energy required to obtain the peak cross section for a triton beam on a deuterium target is $E_{\text {incident }}=165 \mathrm{keV}$.

By setting $\mathrm{F}_{\mathrm{e}}=1$ a minimum electron density is found to be $n_{e}=2.6 \times 10^{32} \mathrm{~m}^{-3}$. Electron densities larger than this could yield net fusion energy production.
Given the reported results, a large fusion reaction gain could be possible with $165 \mathrm{keV}$ tritons. For an electron density of $10^{35} \mathrm{~m}^{-3}$ for $\mathrm{D}(-1)$, a gain factor of 2800 is predicted. Thus our preliminary results indicate that if the claimed electron densities in the new phase of hydrogen were achieved in a target, the energy transfer to electrons would be small enough to allow net energy gain. We conclude that net energy production may be possible using a $\mathrm{D}(-1)$ target, if such a target can be synthesized in bulk.

\section{REFERENCES}

1. J.M. Dawson, H.P. Furth, and F.H. Tenney, Physical Review Letters 26, 1156-1160 (1971).

2. M. Sherlock, S.J. Rose, and A.P.L. Robinson, Physical Review Letters 99, 255003 (2007).

3. F. Santini, Nuclear Fusion 46, 225-231 (2006).

4. C.A. Ordonez, Fusion Technology 22, 388-390 (1992).

5. C.A. Ordonez, Non-Neutral Plasma Physics V, AIP CP692, 267-272 (2003).

6. L. Holmlid, European Physical Journal A 48, 11 (2012).

7. P.U. Andersson and L. Holmlid, Physics Letters A 373, 3067-3070 (2009).

8. S. Badiei, P.U. Andersson, and L. Holmlid, Applied Physics Letters 96, 124103 (2010).

9. P.U. Andersson, B. Lonn, and L. Holmlid, Review of Scientific Instruments 82, 013503 (2011)

10. F. Olofson and L. Holmlid, Nuclear Instruments and Methods B 278, 34-41 (2012).

11. C.A. Ordonez, D.R. Bickel, V.C. Venezia, F.D. McDaniel, S.E. Matteson, M.I. Molina, Journal of Nuclear Materials 264,133-140 (1999).
The fusion energy gain factor described here is defined as a ratio of fusion energy production rate to energy loss rate by the ion beam. Only energy lost to target electrons was considered. While there are other mechanisms of loss in the system, energy loss to electrons is by far the dominant loss term in neutron generators. Further, the effect of electron motion was not considered in this work. Such motion is likely to reduce the stopping power of the target and increase the fusion energy gain factor. A gain factor greater than unity describes a situation where the fusion energy produced might be greater than the initial energy of the injected ion beam. The electrons were treated as conduction electrons. The Fermi energy is directly related to the electron density, the minimum value of which was established by evaluating an upper limit on the energy transfer to electrons for $\mathrm{F}_{\mathrm{e}}=1$. The minimum value was found to be $n_{e}=2.6 \times 10^{32} \mathrm{~m}^{-3}$.

A new phase of hydrogen $\mathrm{D}(-1)$ was reported in Refs. [6-10] with an electron density $\mathrm{n}_{\mathrm{e}}=10^{35} \mathrm{~m}^{-3}$. 